\title{
Electron beam requirements for Smith-Purcell backward wave oscillator with external focusing
}

\author{
Vinit Kumar ${ }^{1}$ and Kwang-Je Kim ${ }^{2}$ \\ ${ }^{1}$ Raja Ramanna Centre for Advanced Technology, Indore, MP 452013, India \\ ${ }^{2}$ Argonne Accelerator Institute, Argonne National Laboratory, Argonne, Illinois 60439, USA \\ (Received 20 January 2009; published 24 July 2009)
}

\begin{abstract}
Operation of the Smith-Purcell backward wave oscillator requires a flat electron beam. Without the electron beam focusing, the requirement leads to a very stringent criterion on vertical emittance. In this paper, we discuss a way to relax the criterion by introducing an external focusing.
\end{abstract}

DOI: 10.1103/PhysRevSTAB.12.070703

\section{INTRODUCTION}

The Smith-Purcell (SP) free-electron laser in the terahertz region using a low energy electron beam is a backward wave oscillator (BWO) [1,2]. The schematic of a SPBWO is shown in Fig. 1. Here, a sheet electron beam is shown to be propagating at a height $d$ from the top surface of a reflection grating, with a speed $\beta c$ along the $z$ axis, where $c$ is the speed of light. The grating has grooves of width $w$ and depth $h$ and extends uniformly to the positive and negative $x$ direction. The length of the grating and its period are $L$ and $\lambda_{g}$, respectively. For oscillation, the electron beam current should be higher than a threshold, known as start current. Recently, we have studied the electron beam requirements for a SP-BWO including three-dimensional effects and shown that one requires a flat electron beam in a SP-BWO [3]. The usefulness of sheet electron beam in the context of generation of coherent SP radiation has been emphasized also by other authors $[4,5]$. The requirement on vertical emittance and vertical beam size is very stringent if there is no external focusing present. This is so because the electron beam needs to maintain close distance from the top surface of the grating.

With the help of electron beam focusing, however, the electron beam size can be made small even with a relaxed emittance. Furthermore, the beam size can be maintained over a longer distance. The grating length can thus be increased to reduce the start current requirement. The grating length is increased up to the value for which the phase spread due to the effective energy spread remains small.

In the next section, we describe the stringent criteria to be met by the flat beam in a SP-BWO in the absence of external focusing. We then discuss the focusing of the flat beam by the wiggler magnetic field and also the solenoid magnetic field in Sec. III. We discuss the limits on emittance, energy spread, and grating length that can be tolerated in the presence of external focusing in Sec. IV. An example case is discussed in Sec. V to illustrate how the emittance requirement can be relaxed and focusing can be utilized to improve the performance of SP-BWO. Some additional remarks are presented in Sec. VI. Finally, Sec. VII concludes the paper.
PACS numbers: 52.59.-f, 41.60.Cr, 41.20.-q, 41.85.Ew

\section{REQUIREMENT OF FLAT ELECTRON BEAM WITHOUT FOCUSING}

The start current $I_{s}$ in a SP-BWO, as derived in Ref. [3] is given by

$$
I_{s}=\mathcal{J}_{s} \frac{I_{A} \Delta x}{4 \chi} \frac{\beta^{4} \gamma^{4}}{k L^{3}} e^{2 \Gamma_{0} d},
$$

where $\mathcal{J}_{s}$ is the dimensionless start current, $I_{A}=17 \mathrm{kA}$ is the Alfvén current, $\chi$ is the residue of the singularity associated with the surface mode as defined in Ref. [2], $\gamma$ is electron's energy in units of rest energy, $k=\omega / c=$ $2 \pi / \lambda, \lambda$ is the free-space wavelength of the surface mode, $\Gamma_{0}=k / \beta \gamma$, and $\Delta x$ is the half width of the electron beam in the $x$ direction, which is chosen to be

$$
\Delta x=2 \sqrt{\frac{\lambda L}{4 \pi \beta_{g}}},
$$

as described in Ref. [3]. Here, $\beta_{g}$ is the group velocity of the surface mode in units of $c$. The above expression is obtained assuming that the electron beam size is the same as the radiation beam size at the middle of the grating. The dimensionless start current $\mathcal{J}_{s}$ is 7.685 [2] for the sheet beam geometry if we ignore the attenuation due to finite conductivity of the grating material and also ignore the

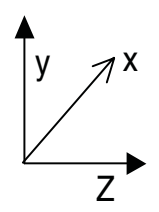

Sheet Electron Beam

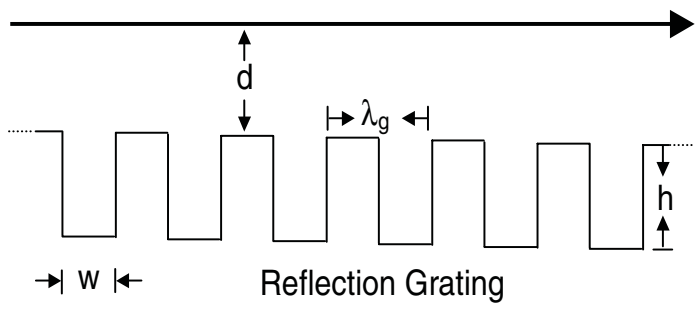

FIG. 1. Schematic of a Smith-Purcell BWO. 
reflection at the ends of the grating. The effect due to finite conductivity of the grating material and the finite reflectivity at the ends of the grating is discussed in Refs. [6,7]. The dependence of start current on $d$ is given by the factor $e^{2 \Gamma_{0} d}$ in Eq. (1) and we therefore choose $d=1 / 2 \Gamma_{0}$. Assuming that the beam edge just touches the grating surface, we require that the half width $\Delta y$ of the electron beam in the $y$ direction is given by

$$
\Delta y=1 / 2 \Gamma_{0} .
$$

When no focusing is present, the unnormalized rms emittance $\epsilon_{y}$ in the vertical direction of the electron beam should satisfy [3]

$$
\epsilon_{y} \leq \frac{(\Delta y)^{2}}{4 L} .
$$

For low electron beam energy and terahertz wavelengths, $\Delta y$ is typically a few tens of microns and $L$ about $1 \mathrm{~cm}$. The emittance should then be less than a few nm-rad, which is a very stringent requirement. Using Eq. (2), the requirement on unnormalized rms emittance in the $x$ direction is shown to be [3]

$$
\epsilon_{x} \leq \frac{\lambda}{4 \pi \beta_{g}} .
$$

The electron beam size and emittance in the $x$ direction are typically required to be in the range of few $\mathrm{mm}$ and few $\mu \mathrm{m}$-rad, respectively. The SP-BWO therefore, in the absence of external focusing, requires a flat beam of emittance ratio around 1000:1.

\section{PRODUCTION AND FOCUSING OF FLAT BEAMS}

We now consider how the emittance requirement can be relaxed by focusing. We discuss two methods of generating and focusing flat electron beams.

\section{A. Focusing of a flat beam using a wiggler}

In the first method, to generate the flat electron beam, as proposed in Ref. [8], a round cathode is placed in a magnetic field and when the electron beam comes out of the magnetic field, it acquires an angular momentum. The electron beam is then passed through a set of three skew quadrupoles to generate a flat electron beam. The flat electron beam generated in this way can be kept focused using a wiggler field, when it skims over the grating top surface.

Low energy electron beams are typically focused using a solenoid magnetic field. However, when a flat electron beam is generated from the angular momentum dominated beam in the field-free region as mentioned above, it starts rotating when it enters the solenoid from the field-free region. The rotation of the beam results from the angular kick due to Lorentz force arising due to interaction of the electron's longitudinal velocity with a radial magnetic field at the entrance of the solenoid. The rotation takes place at the Larmor frequency $\omega_{L}=e B / 2 \gamma m$, where $e$ is the electronic charge and $B$ is the magnetic field inside the solenoid [9]. For a round beam, the rotation may not be of much consequence, but this becomes a serious matter for a flat beam in SP-BWO, where the ratio between the horizontal size and the vertical size is larger than 100 . The vertical electron beam size will increase by a large amount due to rotation of the electron beam. Note that the rotation cannot be compensated by rotating the grating since the beam will be twisted as the amount of rotation will decrease linearly with the distance from the head.

One way of focusing an electron beam in the vertical as well as the horizontal plane without any rotation was first discussed by Scharlemann using a wiggler with a parabolic pole shape [10]. The possibility of focusing a sheet beam using the wiggler magnetic field has also been earlier discussed and demonstrated by Booske et al. [11]. The schematic of using a wiggler for focusing the electron beam in a SP-BWO is shown in Fig. 2. The components of a magnetic field in such a wiggler can be written as

$$
\begin{gathered}
B_{x}=\frac{k_{x}}{k_{y}} B_{w} \sinh k_{x} x \sinh k_{y} y \cos k_{w} z, \\
B_{y}=B_{w} \cosh k_{x} x \cosh k_{y} y \cos k_{w} z, \\
B_{z}=-\frac{k_{w}}{k_{y}} B_{w} \cosh k_{x} x \sinh k_{y} y \sin k_{w} z,
\end{gathered}
$$

where $B_{w}$ is the peak magnetic field in $y$ directions on the $z$ axis, $k_{w}=2 \pi / \lambda_{w}$, and $\lambda_{w}$ is the wiggler period. To satisfy Maxwell equations, we need to impose the condition $k_{x}^{2}+$ $k_{y}^{2}=k_{w}^{2}$.

The envelope equation for a nonaxisymmetric beam in the presence of this magnetic field is given by

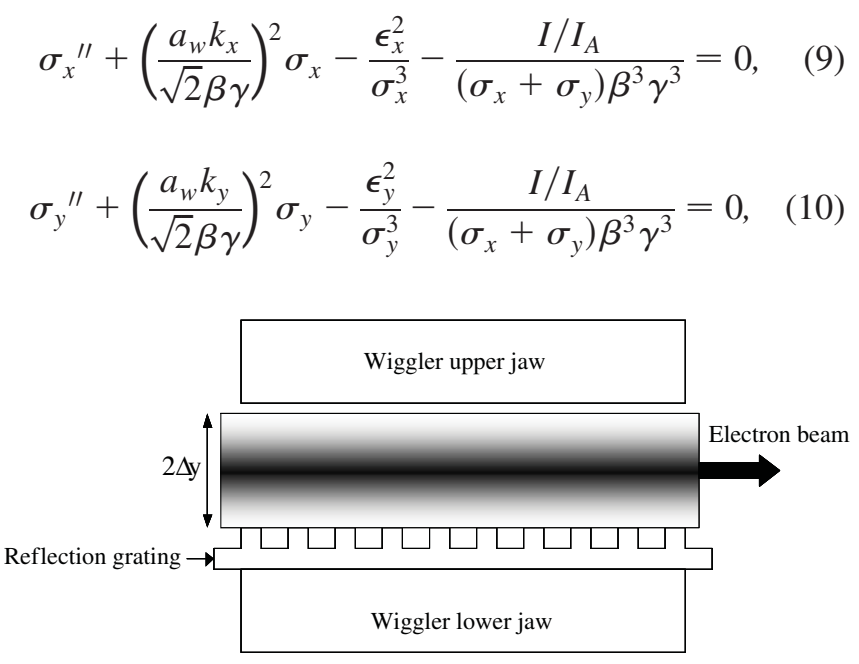

FIG. 2. Schematic of focusing in a Smith-Purcell BWO using wiggler. 
where $\sigma_{x}$ and $\sigma_{y}$ are the rms beam sizes in $x$ and $y$ directions, respectively, $a_{w}=e B_{w} \lambda_{u} / 2 \pi m c$, and $I$ is the electron beam current. Note that the above equation is averaged over undulator period. The space-charge effect has also been taken into account in the above equations. In order to make sure that the space-charge does not deteriorate the emittance, one should make sure that the spacecharge term (fourth term) in these equations is smaller than the emittance term (third term). The wiggler magnetic field provides focusing in the $x$ as well as the $y$ direction as seen from the above equations where the second term specifies the betatron focusing strength. The effective CourantSnyder envelope functions [12] averaged over the wiggler period in the $x$ and $y$ directions are given by

$$
\beta_{x, y}=\frac{\sqrt{2} \beta \gamma}{a_{w} k_{x, y}} .
$$

Neglecting the space-charge effect, the matched electron beam size $\sigma_{x}$ and $\sigma_{y}$ in the $x$ and $y$ directions, respectively, are

$$
\sigma_{x, y}=\sqrt{\beta_{x, y} \epsilon_{x, y}} .
$$

We choose $\sigma_{x}=1 / 2 \Delta x$ and $\sigma_{y}=1 / 2 \Delta y$, where $\Delta x$ and $\Delta y$ are given by Eqs. (2) and (3) such that the electron beam sizes are matched inside the wiggler in both directions. For a given value of $\epsilon_{x}$ and $\epsilon_{y}$, we can find a suitable value of $a_{w}, k_{x}$, and $k_{y}$ such that the above equation is satisfied. Note that in the presence of focusing fields, we do not need to satisfy Eq. (4).

For the typical SP-BWO parameters, the focusing requirement is very strong in the vertical direction and is very weak in the horizontal direction. Neglecting the horizontal focusing, $k_{x}=0$ and $k_{y}=k_{w}$. In view of Eq. (12), by choosing a higher value of $B_{w}$, one can tolerate higher emittance $\epsilon_{y}$ and thus the stringent requirement given by Eq. (4) can be relaxed.

\section{B. Focusing of a flat beam using a solenoid}

It is possible to use a solenoid for focusing a flat beam provided the flat beam is generated in the uniform field region of the solenoid and remains inside the solenoid while skimming over the grating. In this scheme, the cathode and the grating are placed inside the solenoid. One has to use a line cathode where the vertical dimension is very small compared to the horizontal dimension such that the vertical emittance and size are much smaller compared to the horizontal emittance and beam size. Several authors have earlier mentioned about the possibility of using a solenoid magnetic field [13-15] to keep the electron beam focused in the Smith-Purcell system.

The field required to guide a flat beam can be determined by requiring that the Larmor radius is much smaller than the vertical beam size. The transverse velocity can be taken as $c \beta \epsilon_{y} / \sigma_{y}$. This will lead to the following requirement on the axial magnetic field $B(0)$ near the cathode:

$$
B(0) \gg \frac{m c \gamma \beta \epsilon_{y}}{e \sigma_{y}^{2}} .
$$

As an example, if we take $\beta \gamma \epsilon_{y}=10 \times 10^{-8} \mathrm{mrad}, \sigma_{y}=$ $20 \mu \mathrm{m}, B(0)$ should be greater than $4.3 \mathrm{kG}$. Hence, the flat beam can be focused in a solenoid magnetic field of few $\mathrm{kG}$.

However, in the context of a flat beam, one needs to be very careful and make sure that the flat beam does not rotate inside the solenoid. In the ideal case, where the longitudinal magnetic field is uniform (such that the radial component is zero), the electron beam will not rotate. However, in the real case, there will be some nonuniformity in the longitudinal magnetic field that will give rise to beam rotation.

We now estimate the rotation in the beam due to nonuniformity in the on-axis longitudinal magnetic field $B(z)$. Let us assume that the cathode is placed at the center of the solenoid $(z=0)$ where the field is maximum. We can then Taylor expand $B(z)$ around $z=0$ as follows:

$$
B(z)=B(0)+\left.\frac{\partial^{2} B(z)}{\partial z^{2}}\right|_{z=0} \frac{z^{2}}{2},
$$

where we have ignored the higher order terms in $z$. Under the paraxial approximation, the radial component can be expressed as

$$
B_{r}=-\frac{r}{2} \frac{\partial B(z)}{\partial z} .
$$

Using the cylindrical coordinate system and taking the $z$ axis as the solenoid axis, the equation for evolution of $v_{\theta}$ is given by

$$
\gamma m \frac{d v_{\theta}}{d z}=e \frac{r}{2} \frac{d B}{d z}
$$

Here, the variation in the radial coordinate is assumed to be very slow and is neglected here. Integrating this equation under this assumption, we get

$$
\theta(z)=\omega_{L} \frac{z}{v_{z}} \frac{1}{3} \frac{\Delta B(z)}{B(0)},
$$

where $\Delta B(z)=B(z)-B(0)$. The above equation clearly explains that the beam undergoes a rotation in the solenoid field and the amount of rotation is proportional to the nonuniformity in the field over the given distance. Note that if we include the variation in radial coordinate, we get an additional $r$-dependent term in Eq. (17).

Let us now estimate the field uniformity that will be required. For a flat beam having vertical size 100 times smaller than the horizontal beam size, one would like the beam rotation to be less than a few milliradians. As an example, if we take $B(0)=2 \mathrm{kG}, z=25 \mathrm{~mm}$, we require 
$\Delta B(z) / B(0)<0.4 \%$ for $35 \mathrm{keV}$ electron beam if we want the beam rotation to be less than $5 \mathrm{mrad}$.

\section{THE LIMIT ON EMITTANCE, ENERGY SPREAD, AND INTERACTION LENGTH WITH EXTERNAL FOCUSING}

After having discussed the two methods of focusing the flat beam in a SP-BWO, let us now discuss the limit on the maximum possible emittance and energy spread that can be tolerated if we use these focusing techniques. We will also analyze how the deleterious effect due to emittance and energy spread become more prominent when we increase the grating length. In principle, the maximum possible emittance that can be tolerated will depend on the maximum possible focusing strength in the given situation. However, focusing gives rise to a spread in the trajectory angle, and therefore in the $z$ velocity given by

$$
\frac{\Delta v_{z}}{v_{z}} \sim \frac{1}{2} \frac{\epsilon_{y}^{2}}{\sigma_{y}^{2}}
$$

A similar contribution will come from horizontal emittance. The effect of $\Delta v_{z}$ is similar to an effective energy spread. We thus show that the effect of beam emittance in the presence of external focusing can be understood in terms of an equivalent energy spread. Assuming that the maximum energy spread that can be tolerated by SP-BWO corresponds to phase mismatch of $\pi / 2$ between the electrons at the exit of the grating, meaning that $\Delta v_{z} \times$ $L / v_{z}=\lambda_{z} / 4$, we get

$$
\epsilon_{y}<\sigma_{y} \sqrt{\frac{\lambda_{z}}{2 L}}
$$

where $\lambda_{z}=\beta \lambda$ is the period of the surface wave along the $z$ axis.

We can thus summarize the procedure for choosing the optimum focusing strength and emittance as follows: For a given set of parameters for SP-BWO, we first choose vertical beam size as given in Eq. (3) and then we choose the maximum possible focusing strength in Eq. (12) (in the case of wiggler focusing) or Eq. (13) (in the case of solenoid focusing) to maximize the tolerance on vertical emittance, keeping in mind the constraint given by Eq. (19).

With the help of external focusing, we can keep the electron beam focused over a longer distance and the grating length can therefore be increased to reduce the start current. However, there will be an optimum grating length after which the deleterious effect due to energy spread will become more prominent. In order to optimize the grating length, it is important to understand the dependence of start current on energy spread and other parameters, e.g., attenuation due to finite conductivity etc.

The effect of the energy spread on the start current of SPBWO can be calculated analytically. The start current in a
SP-BWO is calculated from the dispersion relation of the surface mode supported by the grating and using the boundary conditions [2]. We assume that the amplitude of the surface mode has $e^{\nu \tau}$ type temporal variation and $e^{\kappa \zeta}$ type spatial variation, where $\tau$ is the dimensionless time and $\zeta=z / L$ is the normalized distance along the grating, as defined in Ref. [2]. If we assume that the input beam has an energy distribution function given by $f_{0}(\eta)$, where $\eta=$ $\left(\gamma-\gamma_{0}\right) / \gamma_{0}$ denotes the electron's relative energy variation with synchronous particle, the dispersion relation is given by [16]

$$
\begin{aligned}
(\nu+\alpha L-\kappa)= & {[i \mathcal{J}+Q(\nu+\alpha L-\kappa)] } \\
& \times \int \frac{1}{(\kappa+i \eta)^{2}} f_{0}(\eta) d \eta,
\end{aligned}
$$

where $\mathcal{J}$ is the dimensionless beam current and $Q$ is the space-charge parameter, defined in Ref. [2]. Here, $\alpha$ is the attenuation coefficient of the surface mode due to finite conductivity of grating material [17]. Above is the generalized form of the dispersion relation obtained previously [2]. Note that, for a monoenergetic electron beam, if we put $f_{0}(\eta)=\delta(\eta)$, we get back the result obtained in Ref. [2]. By putting the given initial energy distribution of the electron beam, we can evaluate the integral on the right side of the above equation and thus introduce the effect of finite energy spread. We assume a step function energy distribution given by $f_{0}(\eta)=1 / 2 \Delta \eta$ for $-\Delta \eta<\eta<$ $+\Delta \eta$ and $f_{0}(\eta)=0$, otherwise. For this energy distribution, we obtain the following dispersion relation, which is cubic in $\kappa$ :

$$
\left(\kappa^{2}+\Delta \eta^{2}-Q\right)(\nu+\alpha L-\kappa)=i \mathcal{J} .
$$

Note that $\Delta \eta$ is related to energy spread $\Delta \gamma m c^{2}$ by the relation $\Delta \eta=k_{0} L \Delta \gamma / \beta^{2} \gamma^{3}$. Here, $m c^{2}$ is the rest mass energy of the electron. For a given value of $\nu$, the above cubic equation gives three possible solutions $\kappa_{1}, \kappa_{2}$, and $\kappa_{3}$ for $\kappa$.

The possible values of $\nu$ are obtained by satisfying three boundary conditions. The first boundary condition is that there is no input field in the surface mode at the exit of the grating $(\zeta=1)$ because in a BWO, the surface mode gets amplified in the direction opposite to that of the electron beam and, hence, the input electric field in the surface mode needs to be specified at the exit of the grating. The second boundary condition implies that there is no microbunching in the electron beam at the entrance to the grating $(\zeta=0)$. The third boundary condition implies that there is no energy-phase correlation in the electron beam at the grating entrance. These boundary conditions lead to the following condition [16]:

$$
\begin{aligned}
\left(\kappa_{1}^{2}-Q\right)\left(\kappa_{2}-\kappa_{3}\right) e^{\kappa_{1}} & +\left(\kappa_{2}^{2}-Q\right)\left(\kappa_{3}-\kappa_{1}\right) e^{\kappa_{2}} \\
& +\left(\kappa_{3}^{2}-Q\right)\left(\kappa_{1}-\kappa_{2}\right) e^{\kappa_{3}}=0 .
\end{aligned}
$$


The above equation is the same as obtained earlier in Ref. [2] for the case of a monoenergetic electron beam. The effect of the finite energy spread is seen only in the dispersion relation [Eq. (21)]. For a given $\mathcal{J}, Q$, and $\Delta \eta$, the above equation is a transcendental equation in $\nu$ since $\kappa_{1}, \kappa_{2}$, and $\kappa_{3}$ are functions of $\nu$ through Eq. (21). One can then solve the above equation numerically. We find that there exists a threshold value $\mathcal{J}_{s}$ above which the real part of $\nu$ is positive. This dimensionless start current $\mathcal{J}_{s}$ is a function of $\Delta \eta$.

The above two equations can be used to find out the dimensionless start current $\mathcal{J}_{s}$ as a function of $Q, \Delta \eta$, and $\alpha L$. Using this approach, we calculate the dimensionless start current as a function of energy spread as shown in Fig. 3. This is a universal curve valid for SP-BWO, independent of any parameter. Note that here we have taken $Q=0$ and $\alpha=0$ since we want to emphasize the dependence on $\Delta \eta$. We find that, as the energy spread increases, the start current increases. For different values of $\alpha L$, we can get a family of curves. The important thing to be noted in Fig. 3 is that in order that the start current is not increased significantly due to energy spread, we require that $\Delta \eta<2$. This leads to the condition $\Delta v_{z} \times L / v_{z}<$ $\lambda_{z} / \pi$, which is approximately the same as mentioned earlier in this section based on qualitative arguments. When we increase the grating length, $\alpha L$ also increases resulting in a further rise in $\mathcal{J}_{s}$. The detailed information regarding the dependence of $\mathcal{J}_{s}$ on $\Delta \eta$ and $\alpha L$ that we have obtained

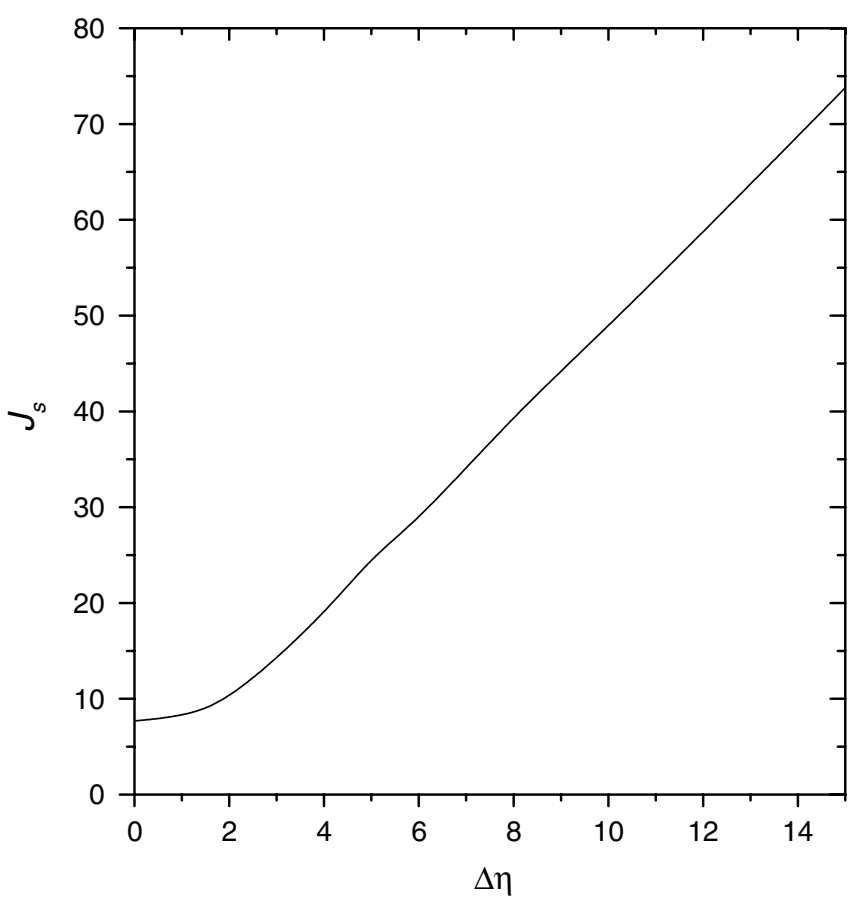

FIG. 3. Dimensionless start current $\mathcal{J}_{s}$ as a function of energy spread $\Delta \eta$, for $Q=0$ and $\alpha L=0$. Here, reflections at the ends of the grating are ignored. in this section can be used to optimize the parameters of SP-BWO.

\section{AN EXAMPLE CASE}

We now discuss an example case for a practical SPBWO. We start with the parameters mentioned in Ref. [3]. The electron beam energy is taken as $35 \mathrm{keV}$, corresponding to $\beta=0.352(\beta \gamma=0.376)$. For grating, we take $\lambda_{g}=$ $173 \mu \mathrm{m}, \quad L=19.03 \mathrm{~mm}, \quad w=110 \mu \mathrm{m}, \quad$ and $h=$ $130 \mu \mathrm{m}$. For these parameters, we found in Ref. [3] that $\lambda=761 \mu \mathrm{m}, v_{g}=0.184 c$, and $\chi=5.83$ per mm. Using Eqs. (2) and (3), we find that $\Delta x=5 \mathrm{~mm}$ and $\Delta y=$ $22.6 \mu \mathrm{m}$. The start current can be determined using Eq. (1). The dimensionless start current $\mathcal{J}_{s}$ is a function of $\alpha L$. Choosing the material of the grating as copper with a DC conductivity of $5.76 \times 10^{7} \Omega \mathrm{m}$, and the electron relaxation time of $2.4 \times 10^{-14} \mathrm{~s}$, it can be shown that $\alpha L=0.59(1-i)$ for the surface mode supported by the above grating parameter. The calculation of $\alpha$ is done following the procedure described in Ref. [17]. For this value of $\alpha L$, it can be shown that $\mathcal{J}_{s}=10.67$. The calculation of $\mathcal{J}_{s}$ including the effect of attenuation of surface mode is discussed in Ref. [6]. Putting these numbers in Eq. (1), we obtain $I_{s}=37.2 \mathrm{~mA}$.

If we do not use any external focusing, the normalized emittance $\left(\beta \gamma \epsilon_{y}, \beta \gamma \epsilon_{x}\right)$ in the $y$ and $x$ directions are required to be smaller than $2.5 \times 10^{-9} \mathrm{mrad}$ and $120 \times 10^{-6} \mathrm{mrad}$, respectively, as calculated from Eqs. (4) and (5).

Let us now find out how much we can relax the vertical emittance requirement using wiggler focusing. Let us explore if we can use the round beam having normalized emittance $\beta \gamma \epsilon_{I}=1 \times 10^{-6}$ mrad that can be easily generated. The flat beam can be generated from this round beam by using round to flat beam transformation [8]. Under this transformation, $\epsilon_{I}=\sqrt{\epsilon_{x} \epsilon_{y}}$. We will choose the ratio of horizontal and vertical emittance 100:1. Such a ratio has been already achieved experimentally [18]. In this case, $\beta \gamma \epsilon_{y}=10^{-7} \mathrm{mrad}$ and $\beta \gamma \epsilon_{x}=10^{-5} \mathrm{mrad}$. Next, we discuss about the wiggler field. Using a hybrid wiggler, with period as small as $2.7 \mathrm{~mm}$ and gap of $1 \mathrm{~mm}$ between the jaws of the wiggler, it is possible to generate a peak field as high as $5 \mathrm{kG}$ as discussed in Ref. [19]. We choose $B_{w}=4.7 \mathrm{kG}$ for which the matched beam size $\sigma_{y}$ comes to be $22.6 \mu \mathrm{m}$, assuming $\beta \gamma \epsilon_{y}=10^{-7} \mathrm{mrad}$. Note that with this value of $\epsilon_{y}$ and $\sigma_{y}$, the criterion given by Eq. (19) is satisfied. The rms beam size in the $y$ direction is twice compared to what we should have opted using Eq. (3). To avoid an increase in the start current, we will increase the grating length by 2 times by choosing $L=$ $38.06 \mathrm{~mm}$. Even with the increased grating length, we find that the constraint on $\epsilon_{y}$ given by Eq. (19) is satisfied. The attenuation factor $\alpha L$ increases by 2 times and we get $\alpha L=1.18(1-i)$. For this value of $\alpha L, \mathcal{J}_{s}=14.43$ [6]. 


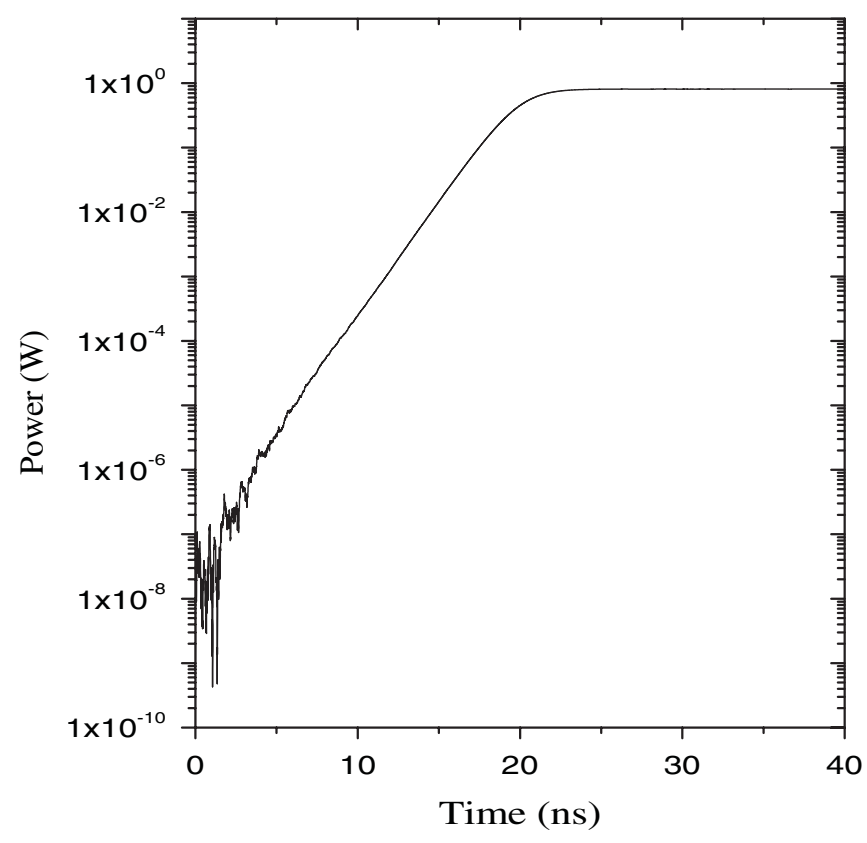

FIG. 4. Evolution of power in SP-BWO for the parameters mentioned in the text. The electron beam current is assumed to be $30 \mathrm{~mA}$.

For this case, $\Delta x$ increases by $\sqrt{2}$ times and we take $\Delta x=$ $7 \mathrm{~mm}$. Putting all these in Eq. (1), we find that the start current reduces to $24 \mathrm{~mA}$. The buildup of power for an electron beam current of $30 \mathrm{~mA}$ is shown in Fig. 4, where calculations have been done using nonlinear numerical simulation [2]. It is seen that the power saturates at around $1 \mathrm{~W}$. Note that it takes around $20 \mathrm{~ns}$ for the power to reach the saturation level starting from noise. The requirement of energy spread can be obtained by requiring $\Delta \eta<2$. This leads to the condition that $\Delta \gamma / \gamma<0.5 \%$. For a DC gun, the energy spread is generally much smaller.

Next, we discuss how the above beam parameters can be achieved using a line cathode immersed in the solenoid field that we discussed in Sec. III B. We assume elliptically shaped, planar, thermionic cathode with major (minor) axis of $\Delta x_{c}\left(\Delta y_{c}\right)$. In order to produce a flat beam with half width $\Delta x$ in the $x$ direction and $\Delta y$ in the $y$ direction, we choose $\Delta x_{c}=\Delta x$ and $\Delta y_{c}=\Delta y$. Hence, we choose a line cathode of $\Delta x_{c}=7 \mathrm{~mm}$ and $\Delta y_{c}=45.2 \mu \mathrm{m}$. The thermal rms normalized emittances for this cathode are given by $\left(\gamma \beta \epsilon_{x}, \gamma \beta \epsilon_{x}\right)=0.5\left(\Delta x_{c}, \Delta y_{c}\right) \sqrt{\frac{k_{B} T}{m c^{2}}}$, where $k_{B}$ is Boltzmann's constant and $T$ is the cathode temperature. For $T=2500 \mathrm{~K}$, we get $\gamma \beta \epsilon_{x}=2.3 \times 10^{-6} \mathrm{mrad}$ and $\gamma \beta \epsilon_{y}=1.5 \times 10^{-8} \mathrm{mrad}$, which are acceptable. The current density at the cathode, corresponding to the current of $30 \mathrm{~mA}$, is around $3 \mathrm{~A} / \mathrm{cm}^{2}$, which is within the range of tungsten cathodes. The magnetic field in the solenoid to guide such a flat beam is much greater than $0.5 \mathrm{kG}$, as calculated from Eq. (13). Hence, we choose a solenoid field of $2.5 \mathrm{kG}$ to guide the beam. The Larmor radius corresponding to these parameters for $2.5 \mathrm{kG}$ field is around $4.5 \mu \mathrm{m}$, which is significantly smaller than the rms beam size $\sigma_{y}=22.6 \mu \mathrm{m}$. Using Eq. (17), we find that the field uniformity $\Delta B / B$ required over the grating length of $38.06 \mathrm{~mm}$ comes to be better than $0.2 \%$ for these parameters if we want the beam rotation to be less than $5 \mathrm{mrad}$.

\section{ADDITIONAL REMARKS}

In this paper, we have discussed that the flat electron beam required in a SP-BWO can be kept focused in the interaction region with the help of external focusing. Two methods of focusing have been discussed. The first method assumes that a flat beam is generated in the field-free region, which is later focused using a wiggler field. Another way for focusing such a flat beam is to use periodic permanent magnet (PPM) focusing where one uses an axisymmetric periodic magnetic field as discussed by Carlsten et al. [20]. However, as discussed in Ref. [20], wiggler focusing provides better stability compared to PPM focusing. The second method assumes that the flat beam is generated inside the solenoid magnetic field by immersing a line cathode in the solenoid. The solenoid focusing is simpler, but one needs to be particularly careful about maintaining field uniformity in the solenoid to make sure that the flat beam does not rotate significantly. At higher currents, there could be problems associated with transmission of sheet electron beam using a solenoid magnetic field as has been discussed by earlier authors [11,21], where it has been pointed out that after a certain threshold, an instability is excited due to $\mathbf{E} \times \mathbf{B}$ drift, where $\mathbf{E}$ is the electric field due to the space charge. This instability is manifested in the form of rotation mode or other familiar instabilities like transverse kink, diocotron, and/or filamentation instability. However, recently Nguyen et al. [22] have proposed that if the beam shape is suitably matched to the beam tunnel, or the beam tunnel is matched to the beam shape, the solenoid focusing method can give superior performance compared to PPM focusing.

We would like to point out that in our calculations, the space charge is not significant. As we can see by explicitly evaluating the third and the fourth terms in Eqs. (9) and (10), the space-charge term is much less compared to the emittance term. Also, we can estimate the electric field arising due to image charge induced on the grating surface, which will have a tendency to pull the electron beam. For a beam current of $30 \mathrm{~mA}$ and full beam width $2 \Delta x=$ $14 \mathrm{~mm}, \beta=0.35$, the surface charge density $\sigma_{0}$ comes to be around $20 \mathrm{nC} / \mathrm{m}^{2}$. The normal electric field due to this $\left(\sim \sigma_{0} / 2 \epsilon_{0}\right)$ is estimated to be around $11.3 \mathrm{~V} / \mathrm{cm}$. For a $38.06 \mathrm{~mm}$ long grating, the vertical deflection due to this electric field is estimated to be around $13 \mu \mathrm{m}$. Note that in actual case, the wiggler will provide vertical focusing and the actual vertical displacement due to this electric field will be much less than this. The beam half width in the vertical direction is $\Delta y=45.2 \mu \mathrm{m}$, which will certainly be much more than the vertical deflection. 
When external focusing is used and the beam size is matched, grating length can be increased to reduce the start current. However, when we increase the grating length, the deteriorating effect of energy spread as well as the attenuation due to finite conductivity of the grating material become more prominent. Finite emittance of the electron beam matched in a focusing channel produces an equivalent energy spread given by Eq. (18). The start current decreases due to inverse cubic dependence on grating length in Eq. (1), but at the same time, it increases since $\Delta \eta$ increases with grating length for a given energy spread. The effect of attenuation and energy spread becomes more important as the length is increased. The optimum grating length will therefore be dependent on all these considerations and can be obtained using the analysis discussed in this paper. The focus of this paper is not a detailed optimization study, but the analysis presented here can be very useful when we want to do a detailed optimization study for a practical SP-FEL.

It is important to point out that in the setup that we have discussed in this paper, the electromagnetic energy grows in the form of surface mode. Different ways of outcoupling this energy in the form of freely propagating mode have been discussed in Ref. [2]. The radiative Smith-Purcell modes appearing due to bunching of the electron beam at higher harmonics will not be outcoupled since they will be blocked by the top jaw of the wiggler (in the case of wiggler focusing), or by the windings of the solenoid (in the case of solenoid focusing). However, one can put a second grating with suitable parameters after the first grating such that the bunched electron beam from the first grating gives off enhanced Smith-Purcell radiation in the second grating and in this way, the Smith-Purcell modes can be outcoupled from the system as discussed in Ref. [2].

In this paper, we have discussed the case where the electron beam skims over one reflection grating. The efficiency of the device can be improved by having an identical reflection grating at the top of the electron beam as discussed by other authors [23,24]. The emphasis in this paper has however been on showing that the performance can be improved by applying external focusing and we have assumed a single reflection grating only to simplify the analysis. Our analysis can be certainly extended and will be applicable for the case of dual gratings discussed by these authors.

\section{CONCLUSIONS}

To summarize, we have performed calculations to show that using a hybrid wiggler with a peak magnetic field of $4.7 \mathrm{kG}$, starting from an electron beam having energy of $35 \mathrm{keV}$, relative energy spread better than $0.5 \%$, normalized rms emittance of $1 \times 10^{-6} \mathrm{mrad}$ and beam current of $30 \mathrm{~mA}$, we can generate around $1 \mathrm{~W}$ of $\mathrm{THz}$ power using SP-BWO. Similar performance can be achieved also by using a solenoid having a field of $2.5 \mathrm{kG}$ to focus the electron beam, but in this case the flat beam should be generated using a line cathode. The line cathode followed by a reflection grating should be immersed together in the uniform field region of the solenoid. Use of external focusing is therefore expected to play an important role in the operation of SP-BWOs.

\section{ACKNOWLEDGMENTS}

It is a pleasure to thank an unknown referee for useful suggestions on solenoid focusing.

[1] H. L. Andrews and C.A. Brau, Phys. Rev. ST Accel. Beams 7, 070701 (2004).

[2] V. Kumar and K.-J. Kim, Phys. Rev. E 73, 026501 (2006).

[3] K.-J. Kim and V. Kumar, Phys. Rev. ST Accel. Beams 10, 080702 (2007).

[4] B. K. Skrynnik et al., Telecommun. Radio Eng. 55, 170 (2001).

[5] J. Gardelle and J.T. Donohue, IEEE Trans. Electron Devices 56, 769 (2009).

[6] V. Kumar and K.-J. Kim, in Proceedings of the 2006 FEL Conference, BESSY, Berlin, Germany, p. 67, http:// www.JACOW.org.

[7] V. Kumar and K.-J. Kim, in Proceedings of the 2006 FEL Conference, BESSY, Berlin, Germany, p. 71, http:// www.JACOW.org.

[8] R. Brinkmann, Y. Derbenev, and K. Flottmann, Phys. Rev. ST Accel. Beams 4, 053501 (2001).

[9] M. Reiser, Theory and Design of Charged Particle Beams (John Wiley \& Sons, New York, 1994).

[10] E. T. Scharlemann, J. Appl. Phys. 58, 2154 (1985).

[11] J. H. Booske et al., J. Appl. Phys. 64, 6 (1988).

[12] E. D. Courant and H.S. Snyder, Ann. Phys. (Paris) 3, 1 (1958).

[13] J. T. Donohue and J. Gardelle, Phys. Rev. ST Accel. Beams 9, 060701 (2006).

[14] D. Li et al., Phys. Rev. ST Accel. Beams 9, 040701 (2006).

[15] L. Schachter, J. Appl. Phys. 67, 3582 (1990).

[16] V. Kumar and K.-J. Kim, in Proceedings of the 2008 FEL Conference, Gyeongju, Korea, p. 14, http://www. JACOW.org.

[17] H.L. Andrews et al., Phys. Rev. ST Accel. Beams 8, 050703 (2005).

[18] P. Piot, Y.-E. Sun, and K.-J. Kim, Phys. Rev. ST Accel. Beams 9, 031001 (2006).

[19] V. A. Papadichev and G. V. Rybalchenko, Nucl. Instrum. Methods Phys. Res., Sect. A 407, 419 (1998).

[20] B.E. Carlsten et al., Phys. Rev. ST Accel. Beams 8, 062002 (2005).

[21] J. H. Booske, B. D. McVey, and T. M. Antonsen, Jr., J. Appl. Phys. 73, 4140 (1993).

[22] K. T. Nguyen et al., IEEE Trans. Electron Devices 56, 744 (2009).

[23] H. P. Freund and T. M. Abu-ElfadI, IEEE Trans. Plasma Sci. 32, 1015 (2004).

[24] Y.-Yao Lin and Y.-Chieh Huang, Phys. Rev. ST Accel. Beams 10, 030701 (2007). 